\title{
A developmental study of the effects of visual and auditory interference on a visual scanning task ${ }^{1,2}$
}

\author{
ELEANOR J. GIBSON AND ALBERT YONAS
}

CORNELL UNIVERSITY

The effects of visual and auditory interference on a visual scanning task were compared with children from the third grade and college sophomores. A highly confusable visual context significantly reduced scanning rate for both children and adults, but a highly confusable auditory context, played over earphones, had no effect on either group. There was a significant age interaction with interfering visual context. It seems likely that theories assuming auditory encoding of visually presented graphic items have little predictive value for a scanning task.

In a previous experiment, Gibson \& Yonas (1966) found a significant age difference in the time required to find a visual target, a letter, embedded in a list of other letters. The scanning task was adapted from one devised by Neisser (1963). When the context letters were made highly confusable with the target letter, search time increased at all age levels, showing a strong interfering effect of visual context. In considering the possible reasons for the longer latencies in children, the hypothesis occurred to us that children in the early stages of reading skill tend to articulate vocally to a greater extent than adults, particularly when difficulties are encountered. It might be that in the present task, children were processing the letters during the search with more explicit vocal responses, or naming the target in order to keep attention on it. If this were the case, one might expect that introduction of a confusing auditory background of letter names coming in through earphones would interfere and increase scanning time for children as compared to adults.

Furthermore, recent studies of short-term memory and recognition of visually presented material have held the implication that the visual material is encoded to an acoustic representation (Conrad, 1964; Sperling, 1963). This raises the question whether visual processing of letters involves such encoding even in a scanning stage. Sperling proposed, for example, that one source of information for auditory storage might be the scanning process; it might be that "observers hear themselves make a verbal response as they scan" (p. 27). If this transformation actually occurs in scanning, it might be expected that even adult Ss would show interference effects from a confusing auditory background. In fact, one would surmise that the effects would be at least as great as with visual confusability.

The present experiment thus sought to compare the interfering effects of a highly confusable auditory context with a highly confusable visual one, and also to compare these effects in children and adults.

\section{Method}

The basic task was the same as in the previous experiment, scanning down a list of 30 letter-strings of four letters each, looking for a designated target letter. The letters were $1 / 4$ in.-high capitals typed with an IBM sign typewriter. The typed list was $10 \mathrm{in}$. long. The $S$ was instructed to start at the top of the list and scan downward until he found the target letter, proceeding as rapidly as possible. He was urged to scan downward, skipping no lines. He held a push button in his hand which he pressed when ready to begin scanning. This caused a list to appear and started a Hunter clock-counter. The S immediately started scanning down the list. When he found the target, he pressed the button again, stopping the clock and turning off the lamps. The $\mathrm{E}$ then recorded the time and placed another list in the display box. If $\mathrm{S}$ had failed to find the target, the list was rerun at the end of the series. Five practice trials were given.

Auditory input accompanied the scanning in all conditions. The S wore earphones over which he heard a male voice naming letters at the rate of two per sec. The sound came on as he pressed the starting switch and ceased when he found the target letter and pressed the switch to stop his scan.

The design of the experiment incorporated three conditions, each including 20 trials with 20 different lists.Condition I had a low-visual, low-auditory confusability of context. The target letter (G) was embedded in a context of letters selected for low confusability in the previous experiment. Its position was randomized across and down the 20 lists, with the restriction that it appeared equally often in the four quadrants of the list from top to bottom and never in the same line more than once. The letters heard over the earphones were selected for low acoustic confusability with the target letter $G$. The values were taken from an auditory confusion matrix for letter names obtained by Conrad (1964).

Condition II had low-visual, high-auditory confusability.It was similar to I, with the same target letter and context letters. Twenty new lists were made up as before. However, the letters heard over the earphones in this condition were six letters of high-auditory confusability with $G$.

Condition 111 had high-visual, low-auditory confusability. The letters heard over the earphones were the same as those in Condition I, but the context letters had high visual confusability, the same lists as those run in this condition of the earlier experiment.

The Ss were 18 children who had just completed third grade, and 18 college sophomores. The experiment was run during the summer, making it possible to run each $\mathrm{S}$ in all three conditions. The order in which the conditions were run was counterbalanced over the subjects of each age group to balance practice effects.

\section{Results}

The time-scores were transformed to take account of position of the target in a list, and averaged to determine scanning rate. As in the earlier experiment, linearity of slope over the 30 positions was not perfect, owing to an initial lag in the first few list positions. It was decided, as before, to include all scores in the analysis since the slope was similar for conditions and ages. It may be seen in Fig. 1 that scanning rate was faster for the adults as compared with the children in all three conditions. Analysis of variance showed that the age difference was significant $(\mathrm{p}<.001)$.

Condition III (high-visual, low-auditory confusability) was more difficult than both the other conditions 


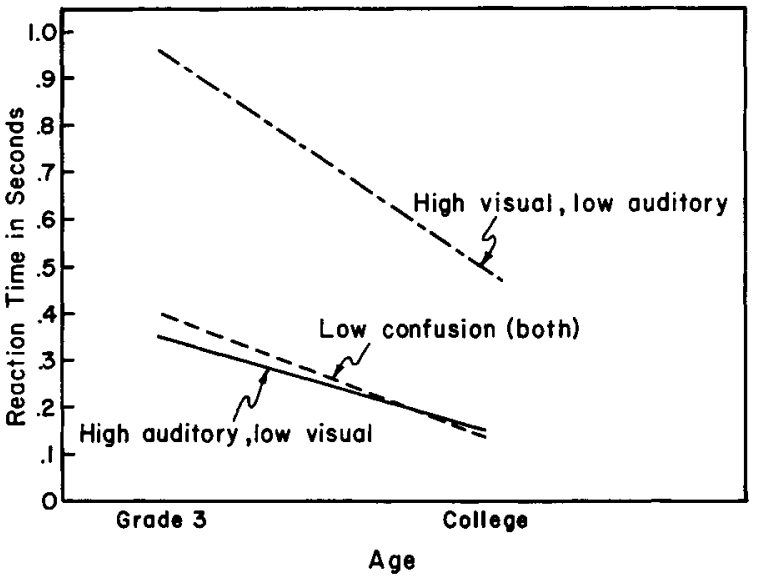

Fig. 1. Mean rate of scan for children and adults on three scanning tasks.

( $p<.001$ ), lowering the scanning rate by $.36 \mathrm{sec}$. for adults and by $.55 \mathrm{sec}$. for children as compared with the control condition of low-visual and low-auditory confusability. On the other hand, the condition of highauditory confusability did not reduce the rate of scan for either the adults or the children. For the children, in fact, speed was slightly (but not significantly) greater with high-auditory confusability.

The interaction of condition with age was significant at the .05 level of confidence. All the variance in this interaction was contributed by the condition of highvisual confusability, which reduced rate of scan relatively more for the children than for the adults. We did not find a similar interaction in the earlier experiment, but since the condition of high-visual confusability always had the benefit of practice in that experiment, it is possible that a small interaction might have been masked.

It is of some interest whether noise as such acted as a distraction, to slow the visual scanning rate. The comparison of the present experiment with the previous one run without auditory noise is not perfect, since practice was not balanced in that experiment, the high-confusion condition always getting the benefit of practice. The rate of scan for adults in the condition of low visual confusability was faster in the present experiment (.14 sec. as compared with .20 sec.), probably due to greater benefit of practice in the present experiment. The rate was very similar in the conditions of high-visual confusability ( .51 in the earlier experiment and .50 in the present one), where practice was comparable. There is thus no evidence that hearing letters spoken while scanning was distracting at all. There was no exactly comparable age group for children in the two experiments.

\section{Discussion}

The strong effect of confusing visual context was confirmed in the present experiment. But attempts to explain the effect on the basis of interfering acoustic representations are discouraged by the total lack of interference from high auditory confusability. These results definitely weaken the hypothesis that visually perceived letters are encoded to acoustic representations as they are scanned. It is possible that such encoding may occur in a recall task, when rehearsal is attempted, but no such strategy appears to be taking place when the task is one of detection.

That information coming in from auditory and visual channels does not combine easily was suggested by an experiment of Broadbent \& Gregory (1965). They presented three visual digits in succession, with a blank interval of $2 / 3 \mathrm{sec}$. between each digit and the next. During each of the blank intervals, a spoken digit was transmitted over a sound channel. Reproduction of the six digits was considerably poorer than when all six digits were presented visually. It is reasonable, in the light of this finding, that interference between auditory and visual channels should not occur, as we found. Letters of high auditory confusability presented over a visual channel could conceivably have a greater interfering effect, and the possibility is being investigated.

\section{References}

Broadbent, D. E., \& Gregory, M. Some confirmatory results on age differences in memory for simultaneous stimulation. Brit. J. Psychol., 1965, 56, 77-80.

Conrad, R. Acoustic confusions in immediate memory. Brit. J. Psychol., 1964, 55, 75-84.

Gibson, E. J., \& Yonas, A. A developmental study of visual search behavior. Percept. \& Psychophys., 1966, 1, 169-171.

Neisser, U. Decision-time without reaction-time: experiments in visual scanning. Amer. J. Psychol., 1963, 76, 376-385.

Sperling, G. A model for visual memory tasks. Hum. Factors, 1963, $5,19-31$.

\section{Notes}

1. This research was supported by NIH Grant MH-07226-02 and by Grant OE6-10-156 from the U. S. Office of Education.

2. We are grateful to Mr. Hart, Principal of the Northeast School of Ithaca, N. Y., for his cooperation in providing laboratory space in the school. 